\title{
Sonographic findings during and after Platelet Rich Plasma injections in tendons
}

\author{
Michele Abate ${ }^{1}$ \\ Sandra Verna ${ }^{2}$ \\ Patrizia Di Gregorio² \\ Vincenzo Salini ${ }^{1}$ \\ Cosima Schiavone ${ }^{1}$
}

1 Department of Medicine and Science of Aging, University G. D'Annunzio, Chieti-Pescara, Chieti Scalo, Italy

2 Immunohaematology and Transfusional Medicine Service, "SS. Annunziata" Hospital, Chieti Scalo, Italy

\section{Corresponding author:}

Michele Abate

Department of Medicine and Science of Aging, University G. D'Annunzio, Chieti-Pescara

Via dei Vestini, 31

66013 Chieti Scalo, Italy

E-mail:m.abate@unich.it

\section{Summary}

Platelet rich plasma has been used in the treatment of tendinopathies, but the sonographic modifications of tendons have received less attention. In this paper we report the results of an ultrasound evaluation, performed during and after plasma injection, in patients with tendinopathy.

The sonographic abnormalities and neovascularization were registered in twenty tendons. Three plasma injections (once a week) were performed, and a rehabilitation program was recommended. Pain and patients' satisfaction were evaluated.

During the injections plasma spread along the collagen fibers, and an intratendineous cleft produced by the injected volume was observed. At 12 months two tendons regained a normal echotexture, while neovessels were absent in seven. The remaining tendons showed less abnormalities and neovascularization in comparison with baseline. The clinical improvement was earlier and more consistent.

The discrepancy between the ultrasound and clinical results may be explained by the peculiar modalities of tendon healing induced by plasma administration.

KEY WORDS: neovascularization, platelet rich plasma, tendinopathy, ultrasound.

\section{Introduction}

The efficacy of Platelet Rich Plasma (PRP) in the treatment of tendinopathies has been proven by several clinical studies ${ }^{1-15}$. However, the sonographic modifications of treated tendons have received less attention, and only in few studies have been specifically addressed, using semiquantitative measures ${ }^{2,16,17}$. Available observations put in evidence that there is only little structural improvement in the long term follow-up, but data about the changes in neovascularization are conflicting. Moreover, only one study has been published about the in vivo spreading of PRP during the injection ${ }^{18}$.

Given the paucity of these informations, in this paper we report a case series of subjects with tendinopathy treated with PRP, where a systematic and semiquantitative sonographic evaluation was performed during the injection and at regular intervals in one year follow-up.

\section{Materials and methods}

This is an open design observational study with a 12 months follow-up period, following basic principles and recommendations in clinical and field science research $^{19}$.

Patients referred to the Echography Unit of our Department for PRP treatment of tendinopathies were enrolled. At baseline, demographic and anthropometric data (sex, age, BMI) were collected. Pain during the previous week, both at rest and during activities, was registered by means of a $0-10 \mathrm{~cm}$ visual analogue scale (VAS).

Before PRP injection, participants underwent an ultrasound (US) and Power Doppler (PD) evaluation of the affected tendon, using a high-resolution, multi-frequency $(6-15 \mathrm{MHz})$ linear array transducer (ProSound ALPHA10, Aloka, Japan). Both longitudinal and transverse scans were taken.

The US criteria adopted for the diagnosis of tendinopathies were the following. The presence of dishomogeneous hypo- or hyperechoic thickening, diffuse or focal, of the tendon, associated with loss of the normal fibrillar pattern and/or irregularity of the tendon margins, was interpreted as sign of degeneration. On the basis of these structural abnormalities, tendons were stratified for severity as "mild" (one area of disorganized echotexture), "moderate" (some areas of disorganized echotexture), and "severe" (disorganized echotexture and diffuse hypo- or hyperechoic areas and/or calcifications) 20

Presence of neovascularization was estimated by means of PD and graded as $(0),(1+),(2++),(3+++)$, 
(4++++), according to the appearance of vessels inside the tendon ${ }^{21}$. To avoid artifacts, sensitivity was optimized for low flow, and the gain was set just below the noise level.

After the diagnostic evaluation, in sterile conditions and under US-guidance, small autologous pure PRP depots were left at several sites into the degenerate tendon areas using a $21 \mathrm{G}$ needle; PRP was placed at the site of most damaged areas, and then proximal and distal, for a total amount of $4 \mathrm{ml}$. No regional anesthetic was used. During the injection, the PRP US distribution pattern into the tendon, and the vessels appearance, before and after the procedure, was evaluated.

A total of 3 injections (once a week) was performed. After the second injection, a rehabilitation program, based on eccentric training and stretching, was recommended daily ( 3 sets $\times 15$ repetitions) at least for 3 months, during which a gradual return to sport activities was encouraged.

The US and clinical assessment was repeated at 3, 6 and 12 months after the last injection, evaluating: a) changes in tendon structures and vascularization; b) pain, both at rest and during activities; c) patients satisfaction (in terms of pain and function) by means of five-points Likert Scale (Not satisfied; Slightly satisfied; Somewhat satisfied; Very satisfied; Extremely satisfied) ${ }^{22}$.

The US scans and the injection procedures were performed by the same well-trained operator (AM).

The study was performed according to the Declaration of Helsinki, and informed written consent was obtained from all the patients.

\section{Statistical analysis}

Data are reported as mean \pm SD for continuous variables, whereas categorical and dichotomous variables are reported as frequencies and percentage. The significance level was determined at $p<0.05$. The two-sample Student's t-test was used to compare continuous variables, when the distribution of data was normal; the Wilcoxon's rank sum test was used otherwise. The $\chi^{2}$ test was used to evaluate associations between categorical data.

\section{Results}

Twenty patients were evaluated (17 males and 3 females; mean age $41.2 \pm 15$ years; range 18-68 years; BMI $24.7 \pm 3.3$ ).

At baseline all tendons (11 Achilles, 5 elbow, and 4 patellar tendons) showed sonographic structural abnormalities: mild alterations were observed in 4 cases, while moderate and severe damage was found in 12 and 4 tendons, respectively (Tab. 1). Intratendinous neovascularization was present in 17/20 tendons, and graded as $(1+),(2++),(3+++),(4++++)$ in $1,8,5$, and 3 cases, respectively (Tab. 2 ).

During the injections a constant feature was the observation of PRP spreading (as hypo/hyperechoic material) along the collagen fibers, also at some centimeters from the tip of the needle, associated to a simultaneous bulging of the whole tendon. An intratendineous cleft was produced by the injected volume and appeared as an anechoic area with irregular mar-

Table 1. Sonographic features of the tendons treated.

\begin{tabular}{|c|c|c|c|c|c|}
\hline & Baseline & 3 months & 6 months & 12 months & $p^{*}$ \\
\hline Normal & 0 & $2(10 \%)$ & $2(10 \%)$ & $2(10 \%)$ & \\
\hline US abnormalities & 20 & $18(90 \%)$ & $18(90 \%)$ & $18(90 \%)$ & ns \\
\hline - $\quad$ Mild & $4(20 \%)$ & $2(11.1 \%)$ & $4(11.1 \%)$ & $8(44.4 \%)$ & ns \\
\hline - Moderate & $12(60 \%)$ & $12(66.6 \%)$ & $12(66.6 \%)$ & $9(50 \%)$ & ns \\
\hline - Severe & $4(20 \%)$ & $4(22.2 \%)$ & $2(22.2 \%)$ & $1(5.5 \%)$ & ns \\
\hline
\end{tabular}

* Baseline versus 12 months

Summing up moderate and severe lesions, the comparison between baseline and 12 months follow-up values shows a significant difference $(16 / 20$ vs $10 / 18 ; p=0.04)$

Table 2. Neovascularization.

\begin{tabular}{|c|c|c|c|c|c|}
\hline & Baseline & 3 months & 6 months & 12 months & $p^{*}$ \\
\hline Absent & $3(15 \%)$ & $3(15 \%)$ & $4(20 \%)$ & $7(35 \%)$ & ns \\
\hline Present & $17(85 \%)$ & 17 (85\%) & $16(80 \%)$ & $13(65 \%)$ & ns \\
\hline$(+1)$ & $1(5.8 \%)$ & $4(23.5 \%)$ & $5(31.2 \%)$ & $8(61.5 \%)$ & 0.001 \\
\hline$(+2)$ & $8(47 \%)$ & $5(29.4 \%)$ & $8(50 \%)$ & $4(30.7 \%)$ & ns \\
\hline$(+3)$ & $5(29.4 \%)$ & $6(35.2 \%)$ & $3(18.7 \%)$ & $1(7.6 \%)$ & ns \\
\hline$(+4)$ & $3(17.6 \%)$ & $2(11.7 \%)$ & 0 & 0 & ns \\
\hline
\end{tabular}

* Baseline versus 12 months

Summing up higher degrees [(+3) and (+4)], the comparison between baseline and 12 months follow-up values shows a significant difference $(8 / 17$ vs $1 / 13 ; p=0.01)$ 
gins. At the end of the procedure neovessels completely disappeared in the region of interest of all the tendons treated (Fig. 1).
At 3, 6, and 12 months after treatment only two tendons (2 elbow extensors) regained a normal echotexture (Fig. 2), while the remaining, albeit less abnor-
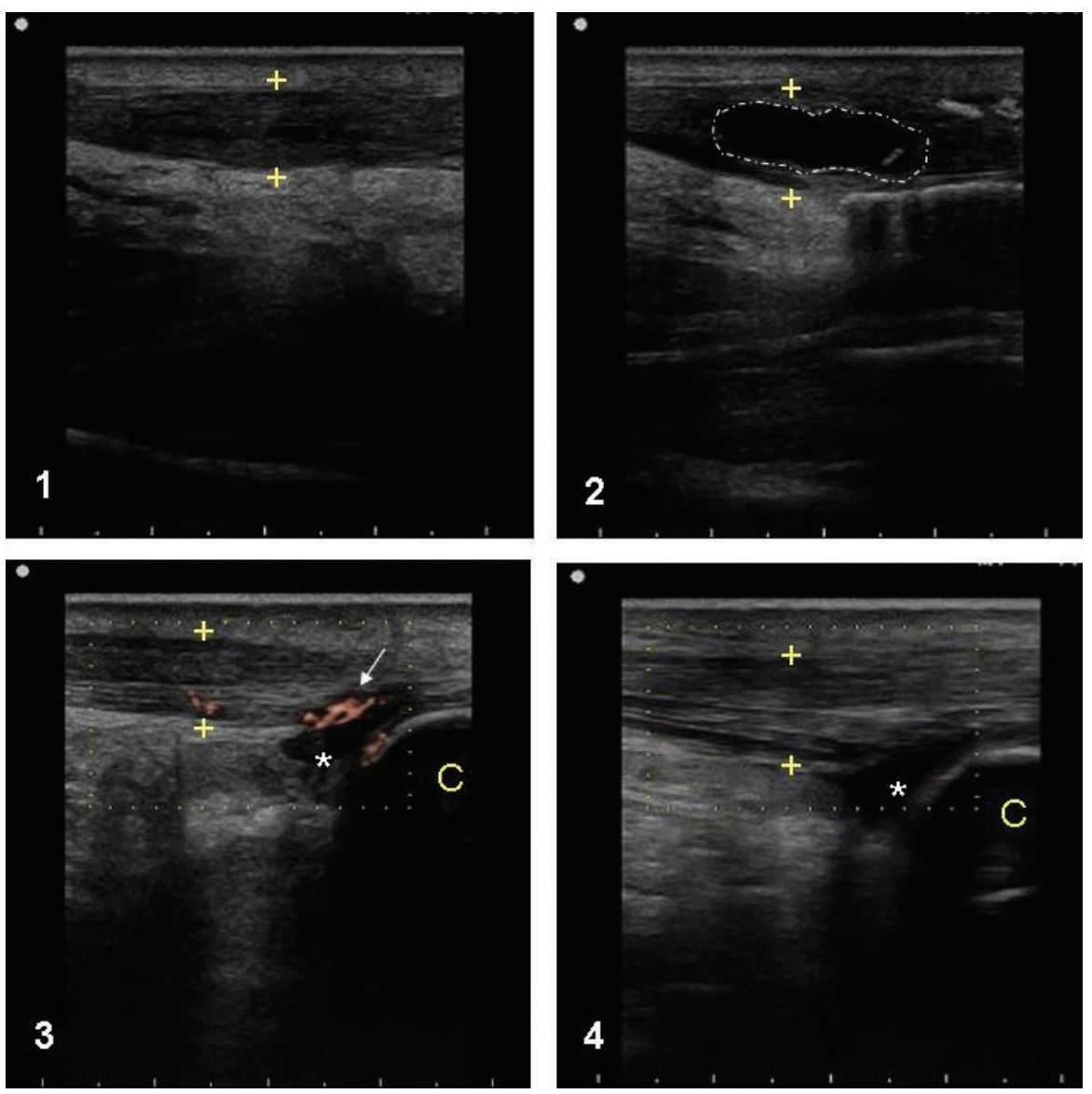

Figure 1. Sonographic findings during, after, and at the end of an ultrasound guided injection for the treatment of Achilles midportion tendinopathy.

Before the procedure, Achilles tendon appears thickened (calipers) and hypoechoic areas are observed at the midportion level (panel 1). Neovessels can be detected in the ventral and distal portion of the tendon (arrow) (panel 3).

During the procedure, the injected volume unsticks the collagen fibers, also at some centimeters from the tip of the needle, and an interstitial cleft (anechoic area) is seen (dot lines); a simultaneous bulging of the whole tendon is observed (calipers) (panel 2). At the end of the injection neovessels completely disappear (panel 4).

$\mathrm{C}=$ Calcaneal bone; * retrocalcaneal bursitis
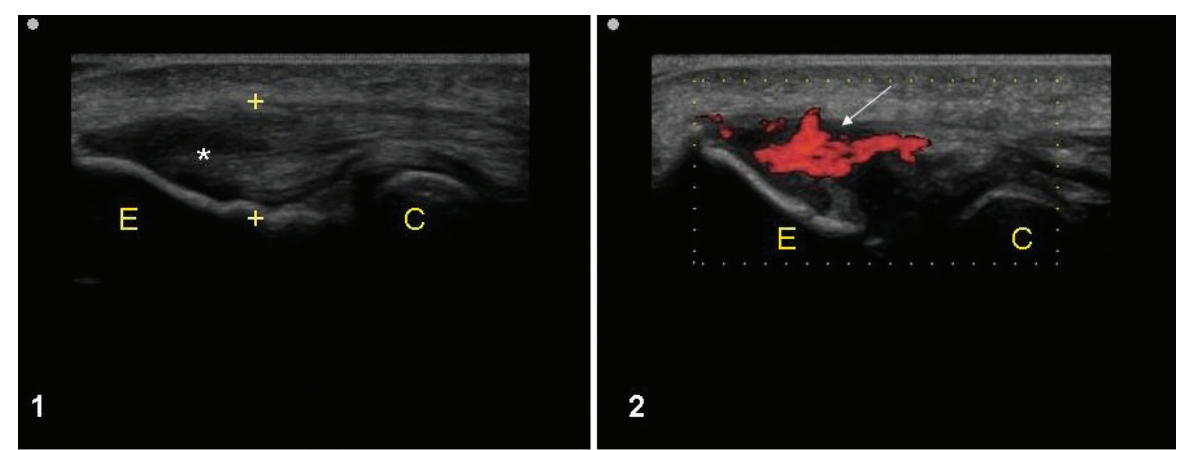

Figure 2. Sonographic improvement in a patient with elbow extensors tendinopathy.

At baseline, the extensor tendons appear thickened (calipers) and hypoechoic $\left.{ }^{*}\right)$ (moderate damage) (panel 1); neovascularization is clearly seen at the insertional level with power doppler $(3+++)$ (arrow) (panel 2).
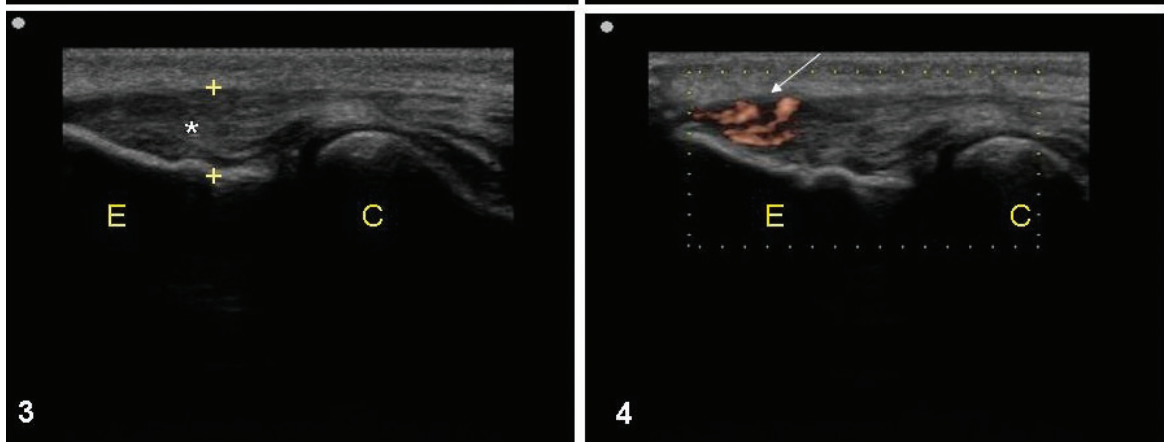

At 12 months follow-up, tendon thickness is reduced and tendon echotexture appears more normal, although some hypoechoic areas are seen at the insertional level $\left(^{*}\right)$ (panel 3); neovessels are reduced (arrow) (panel 4).

$\mathrm{E}=$ Medial Epicondyle; $\mathrm{C}=$ Radial Capitellum 
malities were observed, yet showed US damage. Three Achilles and two patellar tendons did not show any changes. Summing up the cases with severe and moderate US abnormalities, at 12 months, a significant decrease was found; conversely, those with mild and absent US abnormalities increased (Tab. 1).

Likewise, the presence and degree of neovessels decreased during the whole length of the study, and neovascularization was absent in seven tendons at the final follow-up (Fig. 2). The patients with (1+) neovascularization increased significantly, and conversely those with the highest degrees $[(3+++)$ and $(4++++)$ ] decreased $(p=0.01)$ (Tab. 2).

Pain, both at rest and during activities, significantly decreased at 3, 6, and 12 months (Graph 1). At the end of the treatment, 5 patients $(25 \%)$ were extremely satisfied, while 6 (30\%) were very satisfied; the comparison between 3 and 12 months follow-up was statistically significant (Tab. 3).

\section{Discussion}

The present study shows that, after PRP injections, tendon echotexture improves slightly over time. Indeed, after 12 months, 2/20 tendons resumed a normal US pattern, while the remaining revealed a reduc- tion in the US severity score, which reached statistical significance when the cases with moderate and severe abnormalities were considered as a whole. In addition, the degree of intratendineous vascularization significantly decreased. These observations are in agreement with other studies where an assessment of US and PD patterns after PRP injections was performed $4,6,17$. Indeed, a reduction in the number of hypoechoic areas and in the tendon thickness in the mid and long term (6-12 months), with a more regular tendon appearance, was observed $4,6,17$.

It is, however, noteworthy that symptoms (function and pain, both at rest and during activities) improved earlier and more consistently in comparison with the US echo-structure, and that significant improvement was observed also in subjects with persistent sonographic abnormalities. Our hypothesis is that US abnormalities can persist in tendons perfectly healed, even if with scar tissue inside. On the other side, the US method provides only a rough estimation of the subtle morphologic features of the tendon.

Despite no simple rule can be generalized to describe how PRP works for the treatment of tendinopathy, the positive clinical outcomes can be related to the ability of platelets to regulate various healing mechanisms, that is influencing the inflammatory response, modulating angiogenesis, and inducing cell migration and

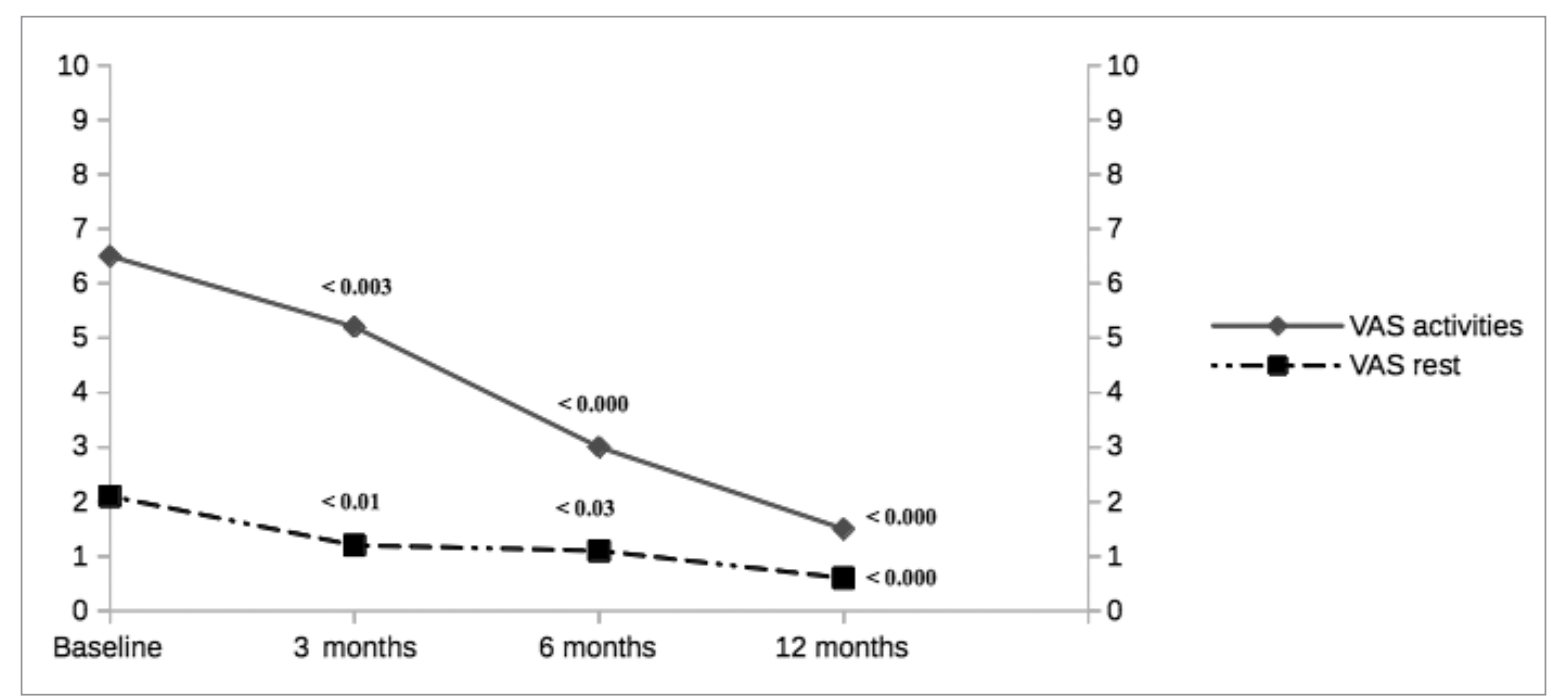

Graph 1. VAS scores, at rest and during activities, in the follow-up periods.

Table 3. Patients' satisfaction during the study periods.

\begin{tabular}{llll}
\hline & 3 months & $\mathbf{6}$ months & $\mathbf{1 2}$ months \\
\hline Not satisfied & $4(20 \%)$ & $3(15 \%)$ & $2(10 \%)$ \\
Slightly satisfied & $7(35 \%)$ & $5(25 \%)$ & $3(15 \%)$ \\
Somewhat satisfied & $6(30 \%)$ & $4(20 \%)$ & $4(20 \%)$ \\
Very satisfied & $3(15 \%)$ & $5(25 \%)$ & $6(30 \%)$ \\
Extremely satisfied & 0 & $3(15 \%)$ & $5(25 \%)$ \\
\hline
\end{tabular}

Summing up the patients very and extremely satisfied, the comparison between 3 months and 12 months is statistically significant $(3 / 20$ vs $11 / 20$ pts; $p=0.008)$. 
proliferation 23,24 . In the short-term, the factors released by the platelets lower inflammation and modulate the pain receptors sensibility, so explaining the positive clinic results; after wards the cell proliferation takes place, with collagen and matrix deposition, and tissue remodeling. Cells usually originate from multipotent stem cells in adjacent tissues, while epitenon tenoblasts, and internal tenocytes contribute to the intrinsic repair process. The final outcome is scar tissue formation, which can partially restore tendon function.

However, discrepancies in the healing processes between tendons are observed. These can be related to the specific functions of tendons themselves. Indeed, weight-bearing tendons, such patellar and Achilles, can be exposed to higher loads (overload), and therefore to an increased strain in comparison to non-load bearing, such elbow extensors, tendons ${ }^{22}$.

These features can explains the apparent discrepancy between the imaging findings and the clinical symptomatology 25 .

As far as the analysis of neovascularisation is considered, the present study shows an evident decrease in the follow-up. This finding is in agreement with other studies $^{6,17}$, but in contrast with the observations of Ferrero ${ }^{4}$ and De Vos $^{16}$, who observed a significant increase after treatment. It is very difficult to explain these discrepancies, which can be related to the release from the platelets both of Vascular Endothelial Growth Factor and of antiangiogenetic factors, which are in turn expressed according to intrinsic and extrinsic influences. In our cases, we hypothise that, beside the complex biochemical machinery, a role could be ascribed to the mechanical effects of the eccentric training, which was performed regularly by the patients. Indeed, it is has been shown in Achilles tendinopathy that the flow in neovessels stops when the ankle joint is in dorsiflexion, whilst in the resting position there is flow again 20,26 . A further explanation could be the neovessels damage due to the mechanical action of injected volume.

Finally, we remark another finding, i.e. the US and $\mathrm{PD}$ changes into the tendon during PRP injections. Indeed, in all tendons, PRP preparation spread along the interfibrillary spaces, also at some centimeters from the tip of the needle, with a simultaneous bulging of the whole tendon. These observations are in agreement with two other studies on PRP diffusion, the first on cadavers ${ }^{27}$, the second in vivo under US control ${ }^{18}$. We think that PRP diffusion can be ascribed to the unsticking of the interfibrillary septa, where the collagen, in chronic tendinopathies, is loose and weak, and therefore more prone to tear. The observation of an anechoic intratendineous cleft during the procedure further support this statement.

The distribution beyond the site of deposition, over a wider region, may eliminate the need for more extensive injections (the so called "peppering technique" $)^{5,6}$. On the other hand, the tracking of the injectate along the tendon allows the sonographer to adjuste the needle position, when he deems that there is excessive dispersion away from the target- ed site, a condition that may cause the treatment failure. Noteworthy, at the end of the procedure, the neovessels completely disappeared in the region of interest of all the tendons treated, probably because of the temporary mechanical compression by the PRP injected nearby.

Some limitations of the present study must be acknowledged. First, a small number of subjects was evaluated, making difficult any consideration about the differences observed between different tendons; second, a control group submitted simply to dry needling was lacking. Indeed, the needling procedure has been proven efficacious by several authors ${ }^{12,28}$, and no difference in echo-structure has been observed after treatment with this technique and PRP injections ${ }^{16}$. Third, a comparison between different PRP compound, i.e. pure PRP versus leukocytes rich plasma, cannot be made. In the present study we used pure PRP because we believe that the presence of leukocytes in the preparation may have a deleterious effect by synthesizing prostaglandins, reactive oxygen intermediates, elastases and other proteases, thus reducing the activity of growth factors and cytokines, with detrimental effects in the quality of the repaired tissue ${ }^{22}$.

In conclusion, our US study during and after PRP injection shows a slight but evident improvement of tendon abnormalities, which, however, are less evident in comparison with the more consistently positive clinical results. In the future, the US modifications should be studied on larger numbers of tendons subcategories (e.g. broad flat versus narrow circular/ ovoid tendons; tendons with a synovial sheath versus tendons within a paratenon) and using PRP preparations with different composition to evaluate possible sonographic differences after treatment.

"This work has not been published previously, it is not under consideration for publication elsewhere, its publication is approved by all Authors and by the responsible authorities where the work was carried out".

\section{Declaration of conflicting interests}

The authors declared no potential conflicts of interests with respect to the authorship and/or publication of this article.

\section{Funding}

The authors received no financial support for the research and/or authorship of this article.

\section{References}

1. Wilson JJ, Lee KS, Miller AT, Wang S. Platelet-Rich Plasma for the Treatment of Chronic Plantar Fasciopathy in Adults: A Case Series. Foot Ankle Spec. 2013. 
2. de Jonge $S$, de Vos RJ, Weir A, et al. One-year follow-up of platelet-rich plasma treatment in chronic Achilles tendinopathy: a double-blind randomized placebo-controlled trial. Am J Sports Med. 2011;39(8):1623-1629.

3. Deans VM, Miller A, Ramos J. A prospective series of patients with chronic Achilles tendinopathy treated with autologousconditioned plasma injections combined with exercise and therapeutic ultrasonography. J Foot Ankle Surg. 2012;51(6): 706-710.

4. Ferrero G, Fabbro E, Orlandi D, et al. Ultrasound-guided injection of platelet-rich plasma in chronic Achilles and patellar tendinopathy. J Ultrasound. 2012;8;15(4):260-266.

5. Gosens T, Den Oudsten BL, Fievez E, van 't Spijker P, Fievez A. Pain and activity levels before and after platelet-rich plasma injection treatment of patellar tendinopathy: a prospective cohort study and the influence of previous treatments. Int Orthop. 2012;36(9):1941-1946.

6. Filardo G, Kon E, Di Matteo B, Pelotti P, Di Martino A, Marcacci M. Platelet-rich plasma for the treatment of patellar tendinopathy: clinical and imaging findings at medium-term follow-up. Int Orthop. 2013;37(8):1583-1589.

7. Vetrano M, Castorina A, Vulpiani MC, Baldini R, Pavan A, Ferretti A.Platelet-rich plasma versus focused shock waves in the treatment of jumper's knee in athletes. Am J Sports Med. 2013;41(4):795-803.

8. Gosens T, Peerbooms JC, van Laar W, den Oudsten BL. Ongoing positive effect of platelet-rich plasma versus corticosteroid injection in lateral epicondylitis: a double-blind randomized controlled trial with 2-year follow-up. Am J Sports Med. 2011;39(6):1200-1208.

9. Thanasas C, Papadimitriou G, Charalambidis C, Paraskevopoulos I, Papanikolaou A. Platelet-rich plasma versus autologous whole blood for the treatment of chronic lateral elbow epicondylitis: a randomized controlled clinical trial. Am J Sports Med. 2011;39(10):2130-2134.

10. Creaney L, Wallace A, Curtis M, Connell D. Growth factorbased therapies provide additional benefit beyond physical therapy in resistant elbow tendinopathy: a prospective, singleblind, randomised trial of autologous blood injections versus platelet-rich plasma injections. Br J Sports Med. 2011;45(12): 966-971.

11. Mishra AK, Skrepnik NV, Edwards SG, et al. Efficacy of Platelet-Rich Plasma for Chronic Tennis Elbow: A DoubleBlind, Prospective, Multicenter, Randomized Controlled Trial of 230 Patients. Am J Sports Med. 2013;12.

12. Rha DW, Park GY, Kim YK, Kim MT, Lee SC. Comparison of the therapeutic effects of ultrasound-guided platelet-rich plasma injection and dry needling in rotator cuff disease: a randomized controlled trial. Clin Rehabil. 2013;27(2):113122.

13. Kesikburun S, Tan AK, Yilmaz B, Yaşar E, Yazicioğlu K. Platelet-rich plasma injections in the treatment of chronic rota- tor cuff tendinopathy: a randomized controlled trial with 1-year follow-up. Am J Sports Med. 2013;41(11):2609-2616.

14. Kaux JF, Crielaard JM. Platelet-rich plasma application in the management of chronic tendinopathies. Acta Orthop Belg. 2013;79(1):10-15.

15. Andia I, Maffulli N. Platelet-rich plasma for muscle injury and tendinopathy. Sports Med Arthrosc. 2013;21(4):191-198.

16. de Vos RJ, Weir A, Tol JL, Verhaar JA, Weinans H, van Schie $\mathrm{HT}$. No effects of PRP on ultrasonographic tendon structure and neovascularisation in chronic midportion Achilles tendinopathy. Br J Sports Med. 2011;45(5):387-392.

17. Finnoff JT, Fowler SP, Lai JK, et al. Treatment of chronic tendinopathy with ultrasound-guided needle tenotomy and platelet-rich plasma injection. PM R. 2011;3(10):900-911.

18. Loftus ML, Endo Y, Adler RS. Retrospective analysis of postinjection ultrasound imaging after platelet-rich plasma or autologous blood: observational review of anatomic distribution of injected material. AJR Am J Roentgenol. 2012;199(4):W501505.

19 Padulo J, Oliva F, Frizziero A, Maffulli N. Muscle, Ligaments and Tendons Journal. Basic principles and recommendations in clinical and field science research. MLTJ. 2013;4:250-252.

20. Abate M, Schiavone C, Salini V, Andia I. Revisiting Physical Examination: Abadie's Sign and Achilles Intratendinous Changes in Subjects with Diabetes. Med Princ Pract. 2013;13.

21. Ohberg $\mathrm{L}$, Alfredson $\mathrm{H}$. Effects on neovascularisation behind the good results with eccentric training in chronic mid-portion Achilles tendinosis? Knee Surg Sports Traumatol Arthrosc. 2004;12(5):465-470.

22. Kolarz G, Kotz R, Hochmayer I. Long-term benefits and repeated treatment cycles of intra-articular sodium hyaluronate (Hyalgan) in patients with osteoarthritis of the knee. Semin Arthritis Rheum. 2003;32(5):310-319.

23. Andia I, Abate M. Platelet-rich plasma: underlying biology and clinical correlates. Regen Med. 2013;8(5):645-658.

24. Ruzzini L, Longo UG, Rizzello G, Denaro V. Stem cells and tendinopathy: state of the art from the basic science to clinic application. Muscles Ligaments Tendons J. 2012;2(3):235-8. Print 2012.

25. Abate M, Di Gregorio P, Schiavone C, Salini V, Tosi U, Muttini A. Platelet rich plasma in tendinopathies: how to explain the failure. Int J Immunopathol Pharmacol. 2012;25(2):325-334.

26. Loppini M, Maffulli N. Conservative management of tendinopathy: an evidence-based approach. Muscles Ligaments Tendons J. 2012;1;1(4):134-137.

27. Wiegerinck Jl, Reilingh ML, de Jonge MC, van Dijk CN, Kerkhoffs GM. Injection techniques of platelet-rich plasma into and around the Achilles tendon: a cadaveric study. Am J Sports Med. 2011;39(8):1681-1686.

28. Nagraba Ł, Tuchalska J, Mitek T, Stolarczyk A, Deszczyński J. Dry needling as a method of tendinopathy treatment. Ortop Traumatol Rehabil. 2013;15(2):109-116. 